\title{
Comparative analysis of the surgical treatment results for multiple myeloma bone disease of the spine and the long bone/soft tissue
}

\author{
JIANGTAO SHEN ${ }^{1}$, XINRU DU ${ }^{2}$, LINGXIU ZHAO ${ }^{3}$, HUI LUO $^{2}$ and ZIYU XU \\ ${ }^{1}$ Department of Orthopedics, Beijing Luhe Hospital, Capital Medical University, Beijing 101100; \\ ${ }^{2}$ Department of Orthopedics, Beijing Chao-yang Hospital, Capital Medical University, Beijing 100020; \\ ${ }^{3}$ Department of Medical Information Center, Beijing Tiantan Hospital, Capital Medical University, Beijing 100050, P.R. China
}

Received August 12, 2017; Accepted February 1, 2018

DOI: $10.3892 / 01.2018 .8559$

\begin{abstract}
The present retrospective study was designed to compare the pain relief, surgery duration, life quality, survival time and relative prognostic factors in multiple myeloma (MM) bone disease patients with different surgical sites. A total of 65 cases were enrolled and divided into two groups. Group A included patients with lesions located in the spine, while Group B included patients with lesions located in the long bone or soft tissue. Pain relief was measured by the visual analogue scale (VAS), neurological impairment was determined according to Frankel classification, and survival was assessed by the Kaplan-Meier method. Cox regression analysis was also used to estimate the effect of factors on the prediction of survival. The hospitalization time, preoperative duration of symptoms, method of surgery, complications, recurrence and survival time were evaluated and compared retrospectively. Pain relief and improvement of life quality were observed in all the patients in groups A and B. No significant differences were detected for the majority of parameters compared between groups A and B, with the exception of the surgery duration, as well as the postoperative VAS score at 1 and 6 months after surgery. The multivariate Cox regression analysis revealed several risk factors significantly associated with survival, including the preoperative VAS score, postoperative chemotherapy, prothrombin time activity (PTA), albumin, lactate dehydrogenase and urine protein level. In conclusion, surgical treatment was an effective therapeutic method in patients with MM. Postoperative analgesic use should be individualized according to the different surgical sites and postoperative periods. Furthermore, preoperative pain, PTA, albumin, urine protein level and postoperative chemotherapy are associated with prognosis.
\end{abstract}

Correspondence to: Professor Xinru Du, Department of Orthopedics, Beijing Chao-yang Hospital, Capital Medical University, 8 Gongti South Road, Chao-yang, Beijing 100020, P.R. China

E-mail: duxinru@163.com

Key words: multiple myeloma, surgical treatment, different surgical sites, comparative analysis

\section{Introduction}

Multiple myeloma (MM) is a hematologic malignancy characterized by the development of a destructive and progressive osteolytic bone disease, which is mainly associated with severe bone pain, pathological fractures, osteoporosis, hypercalcemia and spinal cord compression (1). Although there have been numerous significant improvements in the understanding of the pathophysiologic changes of MM, it remains an incurable disease (2). Destructive skeletal-related events (SREs) are the main clinical manifestations in patients with MM (1,3). It was demonstrated that $70-80 \%$ of patients presented with osteolytic bone lesions at diagnosis, and during the course of MM, $>90 \%$ of patients developed lytic lesions (1-5). If no effective treatment was provided, $>50 \%$ of patients with Durie-Salmon (D-S) stage III MM would suffer at least one SRE within 2 years (6). Frequently, one or more vertebral bodies are detected to be affected by vertebral collapse and/or osteolytic lesions, and long bone fractures more commonly occur in the proximal locations of the upper arm and femora (7). In addition, occasionally soft tissue mass appears in extramedullary tissue, resulting in severe pain and reducing the quality of life. In recent years, surgical consultation has been recommended for MM patients with intractable pain, spinal instability and pathological fractures (8); however, the results of the surgery performed on different sites are not definite. To date, no previous studies have conducted a comparative analysis of different surgical sites of MM patients.

To the best of our knowledge, the present study is the first to compare the results of MM patients receiving surgery for lesions located in the spine with those surgically treated for long bone and soft tissue lesions.

\section{Patients and methods}

Patients and specimens. A total of 65 patients diagnosed with MM were recruited in the present study, including 40 males and 25 females with a mean age of 57.23 years (age range, 20-79 years). The participants were consecutively surgically treated in our institution (Beijing Chao-yang Hospital, Capital Medical University, Beijing, China) over a 5-year period (January 2010 to January 2015). Survival time was recorded from the date of surgery to the last follow-up in June 2016. 
Informed consent was obtained from the subjects for participation into the present study. Ethical approval was obtained from The College Research Ethics Committee of Beijing Chao-yang Hospital, Capital Medical University (Beijing, China).

In this study, the cases were divided into two groups. Group A comprised 33 patients (21 males and 12 females; mean age, 58.32 years; age range, 20-79 years) with surgical sites located in the spine, while Group B included 32 patients (19 males and 13 females; mean age, 56.21 years; age range, 44-74 years) whose surgical sites were in the long bone or soft tissue. The 8 soft tissue cases were initially diagnosed with MM at the Department of Hematology, Beijing Chao-yang Hospital, Capital Medical University (Beijing, China), and subsequently soft tissue masses appeared with the progression of the disease. The D-S stage, International Staging System (ISS) stage and type of MM were recorded, and these data are listed in Table I $(9,10)$. Type of MM was determined using the classification system of the European Society for Medical Oncology, according to the type of monoclonal immunoglobulin secreted by multiple myeloma cells (11). Initially, 2 of the patients were assessed at the Department of Orthopedics at Beijing Chao-yang Hospital, Capital Medical University (Beijing, China) due to experiencing severe pain, and were diagnosed with MM subsequent to surgery and chemotherapy based on specimen examination. The remaining 63 patients were diagnosed with MM upon admission, and accepted treatment by surgery and chemotherapy at the Department of Hematology at Beijing Chao-yang Hospital, Capital Medical University (Beijing, China).

Treatments. In group A, 23 patients $(69.7 \%, 23 / 33)$ received chemotherapy prior to surgery, while 27 patients $(84.4 \%$, 27/32) received chemotherapy prior to surgery in group B. The remaining 10 patients in group A and 5 patients in group $\mathrm{B}$ accepted surgical treatment without preoperative medical therapy. A total of 20 patients $(60.6 \%, 20 / 33)$ in group A and 29 patients $(90.6 \%, 29 / 32)$ in group B continued to receive chemotherapy during the postoperative course. The remaining 13 patients in group $\mathrm{A}$ and 3 patients in group B did not accepted further medical treatment due to limited economic capacity or other reasons. The main chemotherapy schedule was PCD (bortezomib + cyclophosphamide + dexamethasone) or PAD (bortezomib + adriamycin + dexamethasone) in the present study, as previously described $(12,13)$. All the cases receiving preoperative or postoperative chemotherapy completed their chemotherapy courses. In addition, all patients were informed of the benefits of pre- or postoperative radiation therapy, however, the patients participating in the current study selected only pre- or postoperative chemotherapy due to limited understanding of the MM disease and their economic capability.

Lesion locations. In group A, the most common location of bone lesions was in the spine (thoracic, 20 cases; lumbar spine, 5 cases; sacrum, 3 cases; lumbar spine and sacrum, 3 cases; thoracic and lumbar spine, 2 cases). In group B, the lesions were located in the long bones and soft tissue (femur, 12 cases; humerus, 7 cases; clavicle, 2 cases; tibia, 2 cases; radial bone, 1 case; soft tissue, 8 cases).

Surgical procedures. The surgical approach and detailed procedure performed were recorded in the surgeon's operative documents. Patients involved by MM were all medically stable for surgery and complied with the selection criteria for surgical intervention, with the exception of 3 patients in group A (lesions located in T7-9, T4-5 and T5, respectively) who were in a serious condition with irreversible neurological impairment when admitted to the hospital. The preoperative condition of these patients was evaluated via X-ray examination, computed tomography (CT), magnetic resonance imaging (MRI) and blood tests, while ultrasound examination was also required in certain cases with soft tissue lesions.

Different surgical techniques were performed according to the sites of lesions and the surgeon's preference. In group A, 24 patients were treated by lesion resection, posterior decompression and dorsal stabilization with pedicle screw systems. In addition, lesions located in the vertebral body were resected as much as possible, and the defect was filled with bone cement (Fig. 1); a total of 3 patients received this treatment. A total of 5 patients were diagnosed with a vertebral body compression fracture, and percutaneous kyphoplasty (PKP) was performed on the lesion levels. There were 3 patients whose lesions were located in the sacrum causing cauda equina compression; of these, 2 cases were treated by lesion resection and reconstruction with bone cement and a pedicle screw system, while radiofrequency ablation, tumor resection and reconstruction with bone cement was performed in the other case. Furthermore, 1 case with a lesion located in the ventral vertebral body of the first thoracic was treated by vertebral body resection and reconstruction with a titanium cage and bone cement, as well as instrumentation with a vertebral body screw through the anterior approach (Fig. 2). In group B, surgical procedures including tumor resection and reconstruction with bone cement, titanium plates and screws were performed in 20 patients. In addition, 1 patient with a lesion located near the proximal humerus was treated by tumor resection and reconstruction with bone cement and intramedullary nailing (Fig. 3). In 2 cases, a lesion in the femoral head was resected, and replacement of endoprosthesis was performed. Furthermore, 1 case with an intertrochanteric fracture was treated by implantation of intramedullary nailing. There were 8 patients whose surgical sites were in the soft tissue (lower limb, 2 cases; upper limb, 2 cases; buttock, 2 cases; groin, 1 case; back, 1 case). Among these 8 cases, tumor resection alone was performed in 6 patients, and the remaining 2 patients were treated with both tumor resection and nerve decompression.

Follow-up and assessments. The follow-up investigation was conducted by phone or out-patient review. The mean follow-up time was 24.7 months (ranging from 3 to 60 months). Neurological impairment was assessed according to the Frankel classification which provided an assessment of spinal cord function and was used as a tool for spinal cord injury (14). It was defined as five grades (Frankel A, B, C, $\mathrm{D}$ and $\mathrm{E}$ ) according to different motor and sensory function following spinal cord injury. Postoperative radiographs were judged based on local tumor recurrence and the stability of instrumentations. The preoperative visual analogue scale (VAS) score (15), as well as the postoperative VAS scores at 
Table I. Common demographics of the enrolled patients.

\begin{tabular}{|c|c|c|c|}
\hline Characteristic & Group A $(n=33)$ & Group B $(n=32)$ & P-values \\
\hline Male: female & $21: 12$ & $19: 13$ & \\
\hline Age $^{\mathrm{a}}$ (years) & $58.3 \pm 12.7$ & $56.2 \pm 8.2$ & 0.429 \\
\hline \multicolumn{4}{|l|}{ D-S stage of MM } \\
\hline I A/B & 0 & 0 & \\
\hline II A/B & 3 & 4 & \\
\hline III A/B & 27 & 26 & \\
\hline Missing information & 3 & 2 & \\
\hline \multicolumn{4}{|l|}{ ISS stage of MM } \\
\hline $\mathrm{I}$ & 2 & 2 & \\
\hline II & 13 & 12 & \\
\hline III & 15 & 16 & \\
\hline Missing information & 3 & 2 & \\
\hline \multicolumn{4}{|l|}{ Type of $\mathrm{MM}^{\mathrm{c}}$} \\
\hline $\operatorname{IgA}-\kappa$ & 5 & 5 & \\
\hline $\operatorname{IgA}-\lambda$ & 2 & 8 & \\
\hline IgG- $\kappa$ & 13 & 5 & \\
\hline $\operatorname{IgG}-\lambda$ & 8 & 7 & \\
\hline $\operatorname{IgD}-\lambda$ & 2 & 3 & \\
\hline Nonsecretory & 0 & 2 & \\
\hline Missing & 3 & 2 & \\
\hline \multicolumn{4}{|l|}{ Preoperative chemotherapy } \\
\hline Yes & 23 & 27 & \\
\hline No & 10 & 5 & \\
\hline \multicolumn{4}{|l|}{ Postoperative chemotherapy } \\
\hline Yes & 20 & 29 & \\
\hline No & 13 & 3 & \\
\hline Hospitalization time $^{\mathrm{a}}$, days & $19.6 \pm 8.2$ & $18.6 \pm 13.4$ & 0.721 \\
\hline Preoperative duration of symptoms ${ }^{\mathrm{a}}$ (months) & $18.4 \pm 16.3$ & $20.5 \pm 17.1$ & 0.623 \\
\hline Surgery duration $^{\mathrm{a}}(\mathrm{min})$ & $180.0 \pm 74.6$ & $119.7 \pm 45.0$ & $<0.001^{\mathrm{b}}$ \\
\hline Peri-operative bleeding ${ }^{\mathrm{a}}(\mathrm{ml})$ & $343.7 \pm 74.1$ & $253.2 \pm 73.0$ & 0.108 \\
\hline Survival time $^{\mathrm{a}}$ (months) & $24.3 \pm 20.2$ & $20.6 \pm 14.4$ & 0.397 \\
\hline Preoperative VAS ${ }^{a}$ (points) & $8.3 \pm 1.2$ & $7.7 \pm 1.9$ & 0.102 \\
\hline VAS at 1 month after surgery ${ }^{a}$ (points) & $5.5 \pm 1.9$ & $3.3 \pm 1.3$ & $<0.001^{\mathrm{b}}$ \\
\hline VAS at 6 months after surgery ${ }^{a}$ (points) & $2.8 \pm 2.5$ & $1.4 \pm 0.6$ & $<0.001^{\mathrm{b}}$ \\
\hline Platelets $^{\mathrm{a}}\left(\mathrm{x} 10^{9} / \mathrm{l}\right)$ & $197.1 \pm 64.7$ & $182.8 \pm 98.3$ & 0.498 \\
\hline Hemoglobin $^{\mathrm{a}}\left(\mathrm{x} 10^{12} / \mathrm{l}\right)$ & $112.0 \pm 21.1$ & $109.8 \pm 30.1$ & 0.736 \\
\hline $\operatorname{Albumin}^{\mathrm{a}}(\mathrm{g} / \mathrm{l})$ & $31.8 \pm 5.0$ & $33.1 \pm 5.5$ & 0.344 \\
\hline Lactate dehydrogenase $^{\mathrm{a}}$ (U/1) & $352.7 \pm 40.4$ & $239.1 \pm 59.5$ & 0.143 \\
\hline Urine protein ${ }^{\mathrm{a}}(\mathrm{mg} / \mathrm{dl})$ & $24.4 \pm 7.6$ & $14.3 \pm 6.6$ & 0.332 \\
\hline
\end{tabular}

${ }^{a}$ Data are shown as the mean \pm standard deviation. ${ }^{\mathrm{b}} \mathrm{P}<0.05$. MM, multiple myeloma; D-S, Durie-Salmon; ISS, International Staging System; VAS, visual analogue scale; ${ }^{\mathrm{T}}$ Type of MM, the classification system of multiple myeloma according to the type of monoclonal immunoglobulin secreted by multiple myeloma cells (13).

1 and 6 months after surgery were retrospectively compared between the two groups.

Statistical analysis. Groups A and B were compared in terms of the age, hospitalization time, preoperative duration of symptoms, surgery duration, peri-operative bleeding, survival time and laboratory examinations, with differences between the two groups assessed by independent sample t-test and correlation analysis. The postoperative complications and mortality rate between groups A and B were analyzed using an $\chi^{2}$-test. The survival time was estimated using the Kaplan-Meier method. Cox regression analysis was used to estimate the effect of 
A

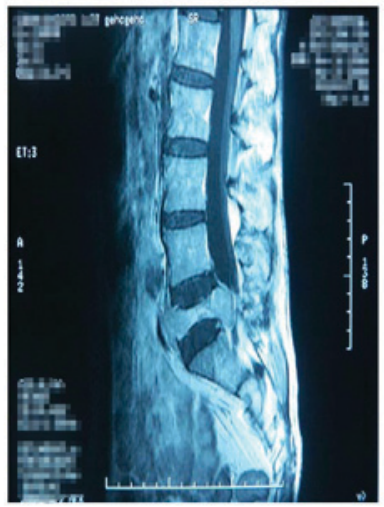

B

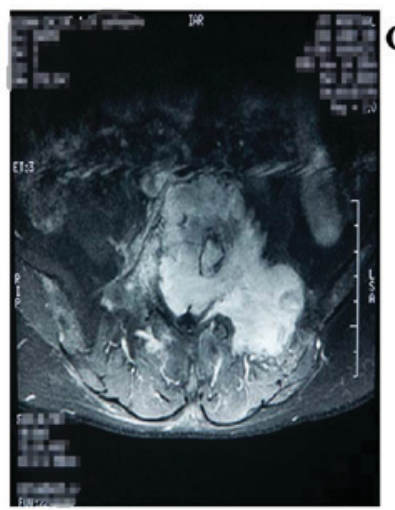

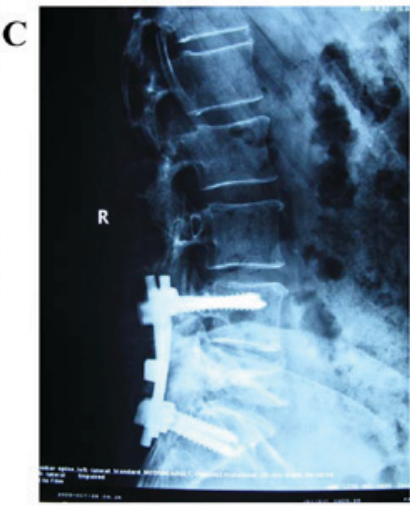

Figure 1. Magnetic resonance imaging and X-ray plain film were used to examine one patient from group A. (A) Magnetic resonance imaging examination revealed that the lesion was located in the spine, the vertebral body of L5 was severely destroyed, and the lumbosacral dural sac was evidently compressed. Image obtained prior to surgery. (B) In this coronary scanning of magnetic resonance imaging examination, lesions were observed to be protruded towards the left and back. Image obtained prior to surgery (C) A postoperative X-ray plain film scan indicated that the fixation devices were implanted successfully. Image obtained following surgery.

A

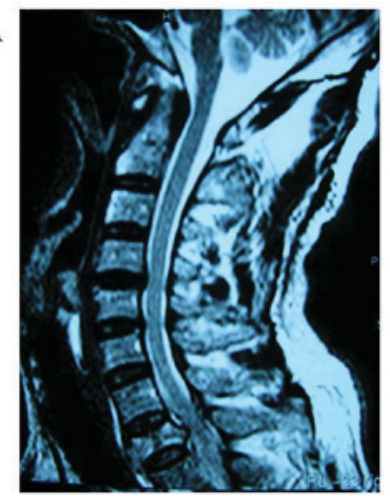

B

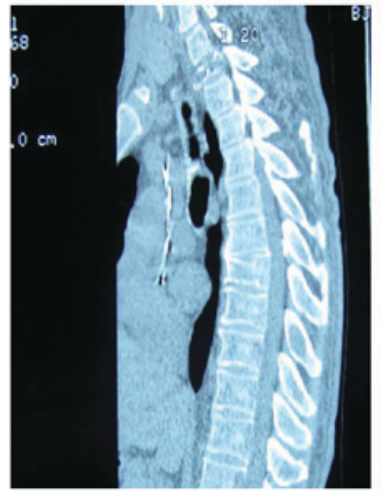

C

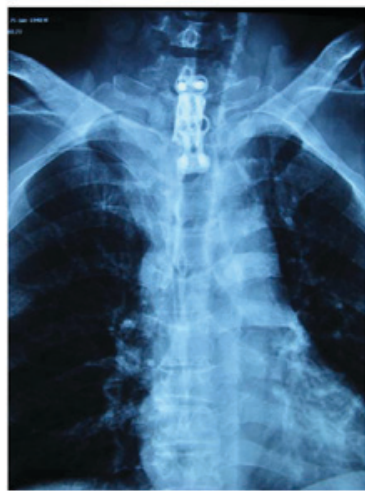

Figure 2. Images from one patient from group A. (A) Magnetic resonance imaging examination demonstrated that the lesion was located in the ventral vertebral body of the first thoracic, and the destroyed part of the vertebral body bulged backward and compressed the dural sac. Image obtained prior to surgery. (B) Computed tomography scan revealed that the majority of the first thoracic vertebrae had been destroyed. Image obtained prior to surgery. (C) The lesion and the destroyed vertebral body were removed via an anterior approach, and were reconstructed with a titanium cage, bone cement and instrumentation with a vertebral body screw. Image obtained following surgery.
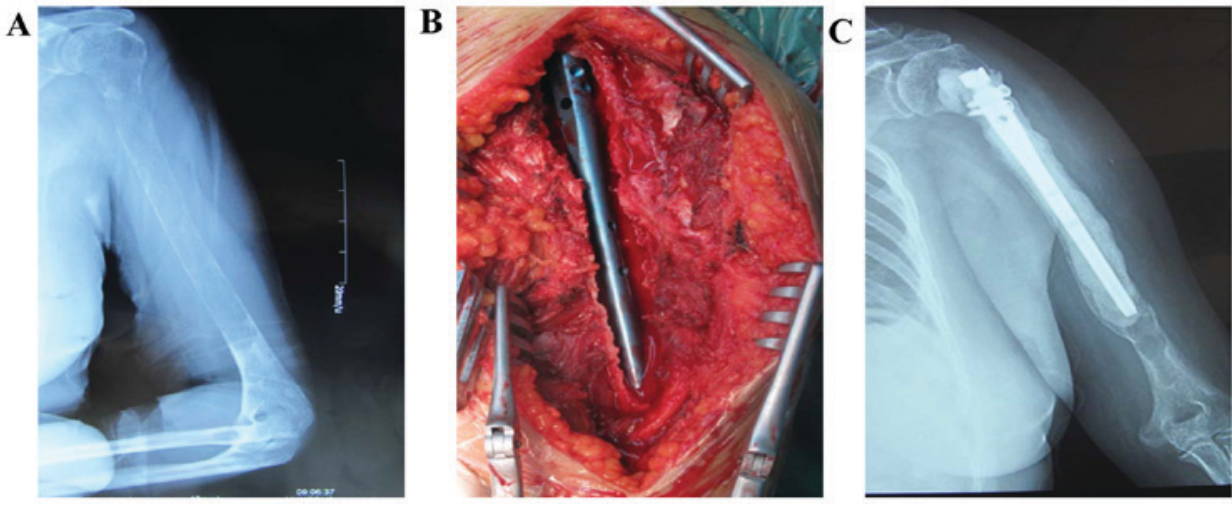

Figure 3. Images from one patient from group B. (A) Myeloma cells invaded in the middle/upper part of the humerus, and the cortical bone became thinner. Image obtained prior to surgery. (B) During surgery, the tumor was removed as much as possible, and proper intramedullary nails were then implanted and fixed with screws. Image obtained during surgery. (C) Postoperative imaging examination demonstrated that the internal fixation devices and bone cement were implanted successfully. Image obtained following surgery.

factors on the prediction of survival. The threshold for a statistically significant difference was set at $\mathrm{P}<0.05$. Statistical analysis was performed with SPSS version 17.0 (SPSS, Inc., Chicago, IL, USA).

\section{Results}

Patient characteristics. The clinicopathological data of patients in groups A and B are presented in Table I. No 
statistical significance was observed in the age, hospitalization time, preoperative duration of symptoms, peri-operative bleeding, survival time, preoperative VAS score, and in the levels of platelets, hemoglobin, albumin, lactate dehydrogenase $(\mathrm{LDH})$ and urine protein between the two groups. However, there was a statistically significant difference in the surgery duration $(\mathrm{P}<0.001)$, as well as in the postoperative VAS scores at 1 and 6 months after surgery (both $\mathrm{P}<0.001$ ) between groups $\mathrm{A}$ and $\mathrm{B}$.

Treatment outcome and survival. In group A, 18 patients succumbed to the disease and 15 patients were alive at the last follow-up, while 14 patients succumbed and 18 were alive in group B. The mortality rate of groups A and B was analyzed by $\chi^{2}$-test, and no significant difference was detected $\left(\chi^{2}=0.552, \mathrm{P}=0.458\right)$. Among the 8 soft tissue cases, 4 patients succumbed and 4 patients were alive at the last follow-up. Pain relief and improvement in the quality of life were obtained in all the patients. The mean VAS scores for the 65 enrolled patients decreased from 7.97 prior to surgery to a value of 4.34 at 1 month after surgery and 2.08 at 6 months after surgery. However, the decrease in the VAS score was significantly greater in group A when compared with that in group B $(\mathrm{P}<0.001$; Table I).

Furthermore, the neurological function improved by different degrees subsequent to the surgical intervention in the majority of patients in group A. Among the $33 \mathrm{MM}$ patients with preoperative neurological dysfunction, 27 patients improved from grade D to E after surgery according to the Frankel classification, while 3 patients improved from Frankel grade $\mathrm{C}$ to $\mathrm{D}$. In addition, 3 patients remained at the same state as that upon admission (Frankel grade C), as their neurological function was already severely and irreversibly impaired, and these patients finally succumbed to the disease at 10, 10 and 23 days after surgery, respectively. In group A, 30 out of the 33 patients $(90.9 \%, 30 / 33)$ demonstrated improvement in neurological impairment following surgery, and no patient developed progressive neurological impairment.

Following surgical intervention, local recurrence was not detected in these patients via associated postoperative imaging examinations, including X-ray plain film, CT and MRI examinations. In group A, 2 patients $(6.1 \%, 2 / 33)$ were complicated with pulmonary infection and 1 case $(3.0 \%, 1 / 33)$ was complicated with septic shock, resulting in a complication rate of $9.1 \%(3 / 33)$ in group A. In group B, only 1 patient $(3.1 \%, 1 / 32)$ was complicated with cerebral infarction, pulmonary infection and urinary infection continuously. The total complication rate in the present study was $6.2 \%(4 / 65)$. In addition, there was no significant difference in the postoperative complications between groups $\mathrm{A}$ and $\mathrm{B}\left(\chi^{2}=0.338\right.$, $\mathrm{P}=0.561$; Table II). The median postoperative survival time in groups A and B was 36 and 60 months, respectively, as determined by the Kaplan-Meier method. When the 8 soft tissue cases were analyzed separately, the median postoperative survival time appeared to be 28 months. The overall survival time of the 8 soft tissue cases was 51.4 months, whereas that of the total 65 cases was 60.2 months. Furthermore, the postoperative 1- and 3-year overall survival rates of group A were 67.2 and $59.5 \%$, respectively, while these were 68.9 and $58.3 \%$, respectively, in group B. The survival curves of the
Table II. Postoperative complications in the two groups.

\begin{tabular}{lccc}
\hline Postoperative complication & Group A & Group B & Total \\
\hline Pulmonary infection & 2 & 0 & 2 \\
Septic shock & 1 & 0 & 1 \\
Cerebral infarction, pulmonary & 0 & 1 & 1 \\
infection and urinary infection & & & \\
Total & 3 & 1 & 4 \\
\hline
\end{tabular}

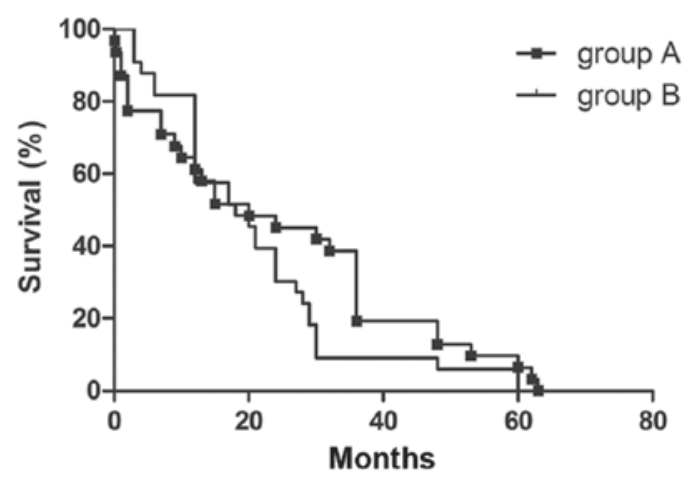

Figure 4. Survival curves of groups A and B of the multiple myeloma patients were compared.

two groups were compared, as shown in Fig. 4. There was no significant difference in mortality rate of groups $\mathrm{A}$ and $\mathrm{B}$ $\left(\chi^{2}=0.552, \mathrm{P}=0.458\right)$.

Risk factors. Multivariate Cox regression analysis revealed the significant survival risk factors, and these included the preoperative VAS score $(\mathrm{RR}=1.731, \mathrm{P}=0.025)$, postoperative chemotherapy $(\mathrm{R} R=5.241, \mathrm{P}=0.048)$, prothrombin time activity (PTA; RR=0.63, $\mathrm{P}=0.008)$, albumin $(\mathrm{RR}=0.586, \mathrm{P}=0.006)$, $\mathrm{LDH}(\mathrm{RR}=1.000, \mathrm{P}=0.037)$ and urine protein level $(\mathrm{R}=1.037$, $\mathrm{P}=0.026$; Table III). Evidence of instrumentation failure and local recurrence was not found in the patients enrolled during the follow-up period.

\section{Discussion}

$\mathrm{MM}$ is the most common primary tumor of the spine, and its typical localization in the vertebral body is in the lower thoracic or lumbar spine (16). Among the SREs secondary to MM, spinal pathologic fractures are considered to be the most common complication (17). However, MM also occurs in the long bone and the soft tissue. Tumor enlargement, pathologic fractures and neurological symptoms are relatively common in MM patients. Apart from treatment approaches including radiotherapy, chemotherapy, bisphosphonates and supportive treatment that are useful $(18,19)$, Dimopoulos et al $(11)$ reported a case with acute bony spinal cord compression and neurological impairment in which the patient was successfully treated with a non-operative approach. However, surgical treatment is also proven to be effective in pain relief and improvement of the life quality for the majority of MM patients with SREs and soft tissue mass. The aim of the present study was to compare 
Table III. Multivariate Cox regression analysis.

\begin{tabular}{lccc}
\hline Parameter & Risk ratio & $95 \%$ confidence interval & P-value \\
\hline Sex & 3.459 & $0.190-62.984$ & 0.402 \\
Age & 0.914 & $0.798-1.048$ & 0.197 \\
Preoperative VAS score & 1.731 & $1.070-2.800$ & $0.025^{\mathrm{a}}$ \\
Hospitalization time & 0.964 & $0.884-1.052$ & 0.409 \\
Preoperative duration of symptoms & 1.086 & $0.976-1.209$ & 0.132 \\
Preoperative chemotherapy & 1.218 & $0.042-35.379$ & 0.908 \\
Postoperative chemotherapy & 5.241 & $1.017-27.014$ & $0.048^{\mathrm{a}}$ \\
Stage (D-S) & & & 0.053 \\
I & 0.000 & $0.000-1.095$ & 0.377 \\
II & 23367 & $0.000-1.142$ & 0.767 \\
III & 2.128 & $0.014-315.35$ & 0.438 \\
Bleeding during operation & 0.999 & $0.995-1.002$ & $0.008^{\mathrm{a}}$ \\
PTA & 0.63 & $0.447-0.886$ & 0.247 \\
PT & 0.205 & $0.014-2.990$ & 0.098 \\
APTT & 0.858 & $0.715-1.029$ & 0.720 \\
TT & 1.112 & $0.621-1.990$ & $0.006^{\mathrm{a}}$ \\
Albumin & 0.586 & $0.401-0.857$ & 0.312 \\
Hemoglobin & 0.966 & $0.904-1.033$ & $0.037^{\mathrm{a}}$ \\
LDH & 1.000 & $0.997-1.003$ & $0.026^{\mathrm{a}}$ \\
Urine protein & 1.037 & $1.004-1.071$ & \\
\hline
\end{tabular}

${ }^{\mathrm{a}} \mathrm{P}<0.05$. VAS, visual analogue scale; D-S, Durie-Salmon; PTA, prothrombin activity; PT, protrombin time; APTT, activated partial thromboplastin time; TT, thrombin time; $\mathrm{LDH}$, lactate dehydrogenase.

MM patients with different presentation sites who were surgically treated.

Based on recent progress $(1,20,17)$, the understanding of the osteoclastogenic and osteoblastic factors involved in the development of myeloma bone disease has improved. The myeloma cells are located adjacent to sites of active bone resorption, which suggests that the mechanism for osteoclastic bone destruction in myeloma bone disease is locally mediated (21). In cases of neurological impairment, radiation therapy and chemotherapy are often effective to diminish the local tumor lesion; however, these strategies do not sufficiently treat spinal instability. It is evident that the combination of surgical and adjuvant treatment is necessary to promote promising outcomes, whether the location of the lesion is in the spine, long bone or soft tissue. Therefore, a primary target in the treatment of MM bone disease is the preservation or restoration of spinal stability, which is similar to the goal in the treatment of metastasis $(22,23)$.

To date, only a few studies have been published reporting a comparative analysis of MM patients with different surgical sites. For instance, Zeifang et al (24) reported that a tumor in long weight-bearing bones was associated with a reduced survival rate as compared with a spinal tumor location (21 vs. 66 months, respectively). However, in the present study, the median survival time of patients with lesions located in the long bone and soft tissue was longer in comparison with that of patients with lesions located in the spine (60 vs. 36 months, respectively), which is not consistent with previous findings reported in the literature. A statistical difference was not evident in MM patients with different anatomical sites of osteolytic bone lesions in the present study. It can be assumed that plasma cells initially infiltrate the axial skeleton, leading to the compression of marrow. With increased cellular proliferation, extensive bone destruction, pathological fractures, hypercalcemia and osteolyses in long weight-bearing bones become evident, indicating an advanced stage of the disease (25). However, in our opinion, the surgery conducted on the spine is a larger invasive procedure compared with procedures on the long bone and soft tissue, which leads to a longer period of time before the patient is able to walk. Thus, it may result in more postoperative complications, including pulmonary infection, deep venous thrombosis and bedsores among others. Finally, patients undergoing surgery on the spine exhibited a shorter median survival time when compared with those undergoing surgery on the long bone and soft tissue. In addition, studies have demonstrated that the presence or absence of extramedullary lesions in MM patients is closely correlated with the prognosis $(26,27)$. The present study revealed that the prognosis of MM patients with extramedullary lesions was worse in comparison with that of patients without extramedullary lesions, which may also explain why the soft tissue cases had a shorter survival time. Other important considerations, including an advanced tumor stage, health condition of the patients, preoperative duration of symptoms, other accompanying diseases and interruption of other treatments, such as chemotherapy, should also be analyzed.

The surgical outcome of lytic bone lesions in MM is frequently compared with that of bone metastases. In earlier 
reports, the overall survival time in metastatic bone disease ranged between 6 and 22 months $(28,29)$, depending on the type of primary tumor. Recent studies concluded that the median survival time of MM patients is longer as compared with that of patients with bone metastases $(24,30)$. This explains the fact that, in myeloma patients requiring orthopedic surgery, a treatment decision should be made comprising a stable reconstruction of the bone defects. Recently, minimally invasive stabilization using bone cement, such as the PKP and percutaneous vertebroplasty (PVP) methods, have been demonstrated to be an effective and safe strategy for vertebral body pathologic fractures in MM patients $(31,32)$. Pain relief was apparent in the early stages following PKP/PVP treatment (20). In the present study, 30 out of the 33 patients $(90.9 \%, 30 / 33)$ in group A exhibited improved neurological impairment subsequent to surgery. However, in a previous study, only 14 out of 49 patients (29\%) exhibited improved neurological function after surgery, and 10 of them were treated by dorsal decompression and stabilization (24). Other authors have reported that up to $81 \%$ of patients with spinal neoplasm experienced neurological improvement following surgery combining anterior-posterior approaches $(33,34)$. The prognosis for neurological recovery is adversely affected by the degree and duration of canal narrowing, demonstrating that patients may benefit from earlier decompression regardless of the selected surgical procedure (35). The surgical sites of the majority of cases included into the present study were in the spine or in the long bone/soft tissue, and patients benefited significantly from surgery. The post-surgical complication rate was low (9.1\% in group A vs. 3.1\% in group B). A study by Pascal-Moussellard et al (36) reported a complication rate of $19 \%$ (17/145) following vertebral metastasis surgery. The complication rates in groups A and B in the current study were lower compared with that reported following surgery in patients with metastases. Refractures in operated limbs were not identified in the present study.

A study including $84 \mathrm{MM}$ patients who were surgically treated reported a recurrence rate of $6 \%$ (24). In the study by Hannisdal et al (25), the total local recurrence rate was $11.1 \%$, which was similar to the recurrence rate of $6-22 \%$ reported in spinal metastases (37-39). In the current study, local recurrence was not reported in any of the 65 patients to date. This may be contributed to the destruction of the MM microenvironment during the surgical procedure and the effect of adjuvant treatment, as well as the limited length of follow-up. Terpos et al (40) reported that, although MRI is superior to positron emission tomography (PET)/CT in the detection of marrow involvement, the PET/CT examination was regarded as the best technique for the follow-up of patients with MM. PET/CT was also proven to be an independent prognostic value at diagnosis and subsequent to treatment. However, in the patients of the present study, only $\mathrm{X}$-ray plain film examination was performed during follow-up and out-patient review due to the financial ability of patients, which should be taken into account. Certain other unknown reasons must also be considered.

Albumin and serum LDH were regarded as markers of the tumor burden and aggressive disease biology, respectively, in the revised ISS classification (41). LDH may be regarded as one of the adjuvant indexes to reflect the prognostic and tumor burden of MM patients (42). In the present study, albumin and $\mathrm{LDH}$ were identified as two of the prognostic factors via multivariate Cox regression analysis. The advanced age, site of lytic bone lesions and D-S stage III were indicated as negative prognostic factors for survival in an earlier study (43). However, no significant difference in these three factors was identified for all the patients and between the two groups in the current study. The selection bias of MM patients and grouping of patients should be considered for this. Besides, although no significant difference in the indication of prognosis was detected for the preoperative duration of symptoms in the present study, this factor serves an important role in improving the quality of life of patients and decreasing complications, such as bone disease, anemia and renal failure in MM (44). General practitioners decision-making aids and public education campaigns are required to reduce the time-to-diagnosis (45). Furthermore, it was observed herein that the VAS score decreased gradually in the two groups between the time prior to surgery and at 1 or 6 months following surgery. Notably, a statistically significant difference was observed in the postoperative VAS score at 1 and 6 months after surgery between groups $\mathrm{A}$ and $\mathrm{B}$ (both $\mathrm{P}<0.001$ ). Thus, it is suggested that the MM patients should be treated individually subsequent to accepting surgery, particularly regarding the postoperative analgesic use. The postoperative pain in MM patients could be controlled effectively by using the appropriate dose of analgesic drugs.

In conclusion, based on the literature and the current findings, it is suggested that surgical treatment is an effective method in MM patients whether the lesion is located in the spine or in the long bone and soft tissue. Preoperative pain, PTA, albumin, urine protein and postoperative chemotherapy are associated with the patient prognosis. Postoperative analgesic use should be individualized according to the different surgical sites and postoperative periods. Finally, studies depicting the outcomes of MM patients with different surgical sites are limited, thus, further investigation need to be undertaken in the future.

\section{Acknowledgements}

Not applicable.

\section{Funding}

No funding was received.

\section{Availability of data and materials}

The datasets generated and analyzed in the present study are included in this published article.

\section{Authors' contributions}

All these authors contributed equally to this work. JTS and XRD conceived and designed the study. LXZ, HL, ZYX and $\mathrm{XRD}$ acquired, analyzed and interpreted the information. JTS and XRD wrote, reviewed and/or revised the manuscript. XRD, ZYX and JTS proofread and formatted the manuscript.

\section{Ethics approval and consent to participate}

Informed consent was obtained from the subjects for participation into the present study. Ethical approval was obtained from 
the The College Research Ethics Committee, Beijing Chao-yang Hospital, Capital Medical University (Beijing, China).

\section{Consent for publication}

Consent for publication of this article has been obtained from all patients included in the study.

\section{Competing interests}

The authors declare that they have no competing interests.

\section{References}

1. Terpos E, Berenson J, Raje N and Roodman GD: Management of bone disease in multiple myeloma. Expert Rev Hematol 7: 113-125, 2014

2. Edwards CM, Edwards JR, Lwin ST, Esparza J, Oyajobi BO, McCluskey B, Munoz S, Grubbs B and Mundy GR: Increasing Wnt signaling in the bone marrow microenvironment inhibits the development of myeloma bone disease and reduces tumor burden in bone in vivo. Blood 111: 2833-2842, 2008

3. Raje N and Roodman GD: Advances in the biology and treatment of bone disease in multiple myeloma. Clin Cancer Res 17: 1278-1286, 2011

4. Terpos E and Dimopoulos MA: Myeloma bone disease: Pathophysiology and management. Ann Oncol 16: 1223-1231, 2005.

5. Vallet $\mathrm{S}$ and Anderson KC: CCR1 as a target for multiple myeloma. Expert Opin Ther Targets 15: 1037-1047, 2011.

6. Coleman RE: Bisphosphonates: Clinical experience. Oncologist 9 (Suppl 4): S14-S27, 2004.

7. Terpos E, Cibeira MT, Blade J and Ludwig H: Management of complications in multiple myeloma. Semin Hematol 46: 176-189, 2009.

8. Adamietz IA, Schöber C, Schulte RW, Peest D and Renner K: Palliative radiotherapy in plasma cell myeloma. Radiother Oncol 20: 111-116, 1991.

9. Greipp PR, San Miguel J, Durie BG, Crowley JJ, Barlogie B, Bladé J, Boccadoro M, Child JA, Avet-Loiseau H, Kyle RA, et al: International staging system for multiple myeloma. J Clin Oncol 23: 3412-3420, 2005.

10. Rajkumar SV, Dimopoulos MA, Palumbo A, Blade J, Merlini G, Mateos MV, Kumar S, Hillengass J, Kastritis E, Richardson P, et al: International Myeloma Working Group updated criteria for the diagnosis of multiple myeloma. Lancet Oncol 15: e538-e548, 2014.

11. Dimopoulos MA and Terpos E: Multiple myeloma. Ann Eur Soc Med Oncol 21 (Suppl 7): vii143-vii150, 2010.

12. He J, Yang L, Han X, Zheng G, Zheng W, Wei G, Wu W, Ye X, Shi J, Xie W, et al: The choice of regimens based on bortezomib for patients with newly diagnosed multiple myeloma. PLoS One 9: e99174, 2014

13. Wang H, Wang L, Lu Y, Chen X, Geng Q, Wang W and Xia Z: Long-term outcomes of different bortezomib-based regimens in Chinese myeloma patients. Onco Targets Ther 9: 587-595, 2016.

14. Zham H, Moradi A, Rakhshan A, Zali A, Rahbari A, Raee M, Ashrafi F, Ahadi M, Larijani L, Baikpour M and Khayamzadeh M: Does Histologic Subtype Influence the Post-Operative Outcome in Spinal Meningioma? Iran J Cancer Prev 9: e3838, 2016.

15. Yasuda T, Kawaguchi Y, Suzuki K, Nakano M, Seki S, Watabnabe K, Kanamori M and Kimura T: Five-year follow up results of posterior decompression and fixation surgery for delayed neural disorder associated with osteoporotic vertebral fracture. Medicine (Baltimore) 96: e9395, 2017.

16. Weinstein JN and McLain RF: Primary tumors of the spine. Spine (Phila Pa 1976) 12: 843-851, 1987.

17. Ha KY, Min CK, Seo JY, Kim YH, Ahn JH, Hyun NM and Kim YC: Bone cement augmentation procedures for spinal pathologic fractures by multiple myeloma J Korean Med Sci 30: 88-94, 2015.

18. Rades D, Huttenlocher S, Dunst J, Bajrovic A, Karstens JH, Rudat $\mathrm{V}$ and Schild SE: Matched pair analysis comparing surgery followed by radiotherapy and radiotherapy alone for metastatic spinal cord compression. J Clin Oncol 28: 3597-3604, 2010
19. Morgan GJ, Child JA, Gregory WM, Szubert AJ, Cocks K, Bell SE, Navarro-Coy N, Drayson MT, Owen RG, Feyler S, et al: Effects of zoledronic acid versus clodronic acid on skeletal morbidity in patients with newly diagnosed multiple myeloma (MRC Myeloma IX): Secondary outcomes from a randomised controlled trial. Lancet Oncol 12: 743-752, 2011.

20. Khan OA, Brinjikji W and Kallmes DF: Vertebral augmentation in patients with multiple myeloma: A pooled analysis of published case series. AJNR Am J Neuroradiol 35: 207-210, 2014.

21. Edwards CM, Zhuang J and Mundy GR: The pathogenesis of the bone disease of multiple myeloma. Bone 42: 1007-1013, 2008.

22. Weber MH, Burch S, Buckley J, Schmidt MH, Fehlings MG, Vrionis FD and Fisher CG: Instability and impending instability of the thoracolumbar spine in patients with spinal metastases: A systematic review. Int J Oncol 38: 5-12, 2011.

23. Fisher CG, DiPaola CP, Ryken TC, Bilsky MH, Shaffrey CI, Berven SH, Harrop JS, Fehlings MG, Boriani S, Chou D, et al: A novel classification system for spinal instability in neoplastic disease: An evidence-based approach and expert consensus from the Spine Oncology Study Group. Spine (Phila Pa 1976) 35: E1221-E1229, 2010.

24. Zeifang F, Zahlten-Hinguranage A, Goldschmidt H, Cremer F, Bernd L and Sabo D: Long-term survival after surgical intervention for bone disease in multiple myeloma. Ann Oncol J Eur Soc Med Oncol 16: 222-227, 2005.

25. Hannisdal E, Kildahl-Andersen O, Grøttum KA and Lamvik J: Prognostic factors in multiple myeloma in a population-based trial. Eur J Haematol 45: 198-202, 1990.

26. Cerny J,FadareO,HutchinsonLandWang SA:Clinicopathological features of extramedullary recurrence/relapse of multiple myeloma. Eur J Haematol 81: 65-69, 2008.

27. Köse M, Buraniqi E, Akpinar TS, Kayacan SM and Tükek T: Relapse of multiple myeloma presenting as extramedullary plasmacytomas in multiple organs. Case Rep Hematol 2015: 452305, 2015.

28. Lahtinen R, Laakso M, Palva I, Virkkunen P and Elomaa I: Randomised, placebo-controlled multicentre trial of clodronate in multiple myeloma. Finnish Leukaemia Group. Lancet (London, England) 340: 1049-1052, 1992.

29. Tatsui H, Onomura T, Morishita S, Oketa M and Inoue T: Survival rates of patients with metastatic spinal cancer after scintigraphic detection of abnormal radioactive accumulation. Spine 21: 2143-2148, 1996.

30. Zadnik PL, Goodwin GC, Karami KJ, Mehta AI, Amin AG, Groves ML, Wolinsky JP, Witham TF, Bydon A, Gokaslan ZL and Sciubba DM: Outcomes following surgical intervention for impending and gross instability caused by multiple myeloma in the spinal column. J Neurosurg Spine 22: 301-309, 2015

31. Zou J, Mei X, Gan M and Yang H: Kyphoplasty for spinal fractures from multiple myeloma. J Surg Oncol 102: 43-47, 2010.

32. Kasperk C, Haas A, Hillengass J, Weiss C, Neben K, Goldschmidt H, Sommer U, Nawroth P, Meeder PJ, Wiedenhöfer B, et al: Kyphoplasty in patients with multiple myeloma a retrospective comparative pilot study. J Surg Oncol 105: 679-686, 2012.

33. Harrington KD: Anterior decompression and stabilization of the spine as a treatment for vertebral collapse and spinal cord compression from metastatic malignancy. Clin Orthop Relat Res: 177-197, 1988.

34. Kluger P, Korge A and Scharf HP: Strategy for the treatment of patients with spinal neoplasms. Spinal Cord 35: 429-436, 1997.

35. Helweg-Larsen S: Clinical outcome in metastatic spinal cord compression. A prospective study of 153 patients. Acta Neurol Scand 94: 269-275, 1996.

36. Pascal-Moussellard H, Broc G, Pointillart V, Siméon F, Vital JM and Sénégas J: Complications of vertebral metastasis surgery. Eur Spine J 7: 438-444, 1998

37. Jansson KA and Bauer HC: Survival, complications and outcome in 282 patients operated for neurological deficit due to thoracic or lumbar spinal metastases. Eur Spine J 15: 196-202, 2006.

38. Weigel B, Maghsudi M, Neumann C, Kretschmer R, Muller FJ and Nerlich M: Surgical management of symptomatic spinal metastases. Postoperative outcome and quality of life. Spine 24: 2240-2246, 1999.

39. Lau D, Leach MR, La Marca F and Park P: Independent predictors of survival and the impact of repeat surgery in patients undergoing surgical treatment of spinal metastasis. J Neurosurg Spine 17: 565-576, 2012. 
40. Terpos E, Dimopoulos MA and Moulopoulos LA: The Role of Imaging in the treatment of patients with multiple myeloma in 2016. Am Soc Clin Oncol Educ Book. Meeting 35: e407-e417, 2016.

41. Rajkumar SV: Myeloma today: Disease definitions and treatment advances. Am J Hematol 91: 90-100, 2016.

42. Long SF, Chen GA and Fang MS: Levels of interleukin-16 in peripheral blood of 52 patients with multiple myeloma and its clinical significance. Int J Clin Exp Med 8: 22520-22524, 2015.

43. Bataille R, Boccadoro M, Klein B, Durie B and Pileri A: C-reactive protein and beta-2 microglobulin produce a simple and powerful myeloma staging system. Blood 80: 733-737, 1992.
44. Kariyawasan CC, Hughes DA, Jayatillake MM and Mehta AB: Multiple myeloma: Causes and consequences of delay in diagnosis. QJM 100: 635-640, 2007.

45. Howell DA, Smith AG, Jack A, Patmore R, Macleod U, Mironska E and Roman E: Time-to-diagnosis and symptoms of myeloma, lymphomas and leukaemias: A report from the Haematological Malignancy Research Network. BMC Hematol 13: 9, 2013. 